\title{
Beneficial Effects of AMP-Activated Protein Kinase Agonists in Kidney Ischemia-Reperfusion: Autophagy and Cellular Stress Markers
}

\author{
Anne-Emilie Declèves ${ }^{a, b, f} \quad$ Kumar Sharma $^{a-c, e} \quad$ Joseph Satriano ${ }^{a, b}$, d, e \\ a Division of Nephrology-Hypertension, O'Brien Kidney Center, ${ }^{b}$ Center for Renal Translational Medicine, \\ 'Institute of Metabolomic Medicine, d'Stein Institute for Research on Aging, e University of California San Diego and \\ the Veterans Affairs San Diego Healthcare System, La Jolla, Calif., USA; ${ }^{f}$ Laboratory of Experimental Nephrology, \\ Faculty of Medicine, Université Libre de Bruxelles (ULB), Brussels, Belgium
}

\section{Key Words}

Mammalian target of rapamycin · Mitophagy · p62/SQSTM1 . PTEN-induced putative kinase-1

\begin{abstract}
Background: Kidney ischemia-reperfusion is a form of acute kidney injury resulting in a cascade of cellular events prompting rapid cellular damage and suppression of kidney function. A cellular response to ischemic stress is the activation of AMP-activated protein kinase (AMPK), where AMPK induces a number of homeostatic and renoprotective mechanisms, including autophagy. However, whether autophagy is beneficial or detrimental in ischemia-reperfusion remains controversial. We investigated the effects of agonist induction of AMPK activity on autophagy and cell stress proteins in the model of kidney ischemia-reperfusion. Methods: AMPK agonists, AICAR $(0.1 \mathrm{~g} / \mathrm{kg})$ and metformin $(0.3 \mathrm{~g} / \mathrm{kg})$, were administered $24 \mathrm{~h}$ prior to ischemia, with kidneys harvested at $24 \mathrm{~h}$ of reperfusion. Results: We observed a paradoxical decrease in AMPK activity accompanied by increases in mammalian target of rapamycin (mTOR) $\mathrm{C} 1$ activity and p62/SQSTM1 expression. These results led us to propose that AMPK and autophagy are insufficient to properly counter the cellular insults in ischemia-reperfusion. Agonist in-
\end{abstract}

duction of AMPK activity with AICAR or metformin increased macroautophagy protein LC3 and normalized p62/SQSTM1 expression and mTOR activity. Ischemia-reperfusion increases in Beclin-1 and PINK1 expressions, consistent with increased mitophagy, were also mitigated with AMPK agonists. Stress-responsive and apoptotic marker expressions increase in ischemia-reperfusion and are significantly attenuated with agonist administration, as are early indicators of fibrosis. Conclusions: Our data suggest that levels of renoprotective AMPK activity and canonical autophagy are insufficient to maintain cellular homeostasis, contributing to the progression of ischemia-reperfusion injury. We further demonstrate that induction of AMPK activity can provide beneficial cellular effects in containing injury in ischemia-reperfusion.

(c) 2014 S. Karger AG, Basel

\section{Introduction}

Acute kidney injury (AKI) refers to a rapid and severe suppression of kidney function frequently associated with high morbidity and mortality. Ischemia-reperfusion (IR) is a common experimental model used to understand the pathogenesis of AKI due to ischemic injury,

\section{KARGER}

E-Mail karger@karger.com

www.karger.com/nee (c) 2014 S. Karger AG, Base

1660-2129/14/1284-0098\$39.50/0 
which can be the result of acute or chronic dysfunction of the heart. IR causes a cascade of cellular events, some prompting DNA and cellular damage leading to cell death and organ dysfunction, while others offer cellular protection. The balance between these responses determines the outcome of IR. A cellular response to ischemia is the activation of $5^{\prime}$ AMP-activated protein kinase (AMPK), which induces a number of cell survival mechanisms. However, it may not be enough to adequately respond to the magnitude of the rapid array of insults the cell faces in this condition. We propose to establish that the protective mechanisms of increasing AMPK activity in IR better prepares the kidney to respond to and manage injury.

AMPK, an evolutionarily conserved serine/threonine kinase, is a central mediator of energy homeostasis responsive to nutritional and metabolic stresses $[1,2]$. AMPK activation provides a protective response which affords mitochondrial protection and biogenesis [3], suppression of extracellular matrix proteins [4], and induction of autophagy. Autophagy would constitute a means to rid the cell of accumulated proteins and organelles damaged by IR injury, such as mitochondria and endoplasmic reticulum, while suppressing apoptosis [5-7].

Cellular stress response mechanism apoptosis, necrosis, and autophagy form a complex network of interrelated features and cross-regulation [8-12], although the mechanisms of this regulation are not well understood. Unlike necrosis, which is a form of cell death in response to external factors, apoptotic cell death is a natural process for aged cells. However, apoptosis can be prompted in response to cellular stressors and can amplify to levels that become unsustainable and injurious to the organ. Apoptosis and necrosis are central components in the extensive loss of tubule epithelial cells observed in IR. AMPK activation offers several mechanisms by which it can aid in the protection [13-16] and biosynthesis [1719] of mitochondria. In addition, it activates a potent mechanism for cell survival and repair, autophagy.

Autophagy, unless otherwise stated, refers to macroautophagy. This form of autophagy entails the formation of an autophagosome vacuole for delivery of its contents to the lysosome for degradation. It is fundamental in ridding the cell of aged and aggregated proteins and is the only known mechanism for disposing of damaged organelles under normal conditions as well as in cells under stress [20-25]. The capacity to sequester aged and damaged cellular components can abate mitochondrial and endoplasmic reticulum-induced apoptosis, thereby allowing the cell to adapt to stress [26]. Suppression of autophagy leads to an accumulation of dysfunctional mito- chondria, aberrant proteins and organelles, promotion of endoplasmic reticulum stress and apoptosis [27], increased oxidative stress with damage to membranes, proteins, and DNA, and genomic instability [28]. Decreased nutrients and increased hypoxia increase AMPK and autophagy during ischemia $[29,30]$. Autophagy can also be increased by a nonnutrient-dependent mechanism(s) during reperfusion via a process associated with reactive oxygen species (ROS) [31], which, in contrast to AMPKinduced autophagy, can become injurious [30, 32, 33]. The role of autophagy in IR is somewhat controversial and may depend upon the source of induction and response mechanism.

IR is characterized by an ischemic phase increase in AMPK activity. During the subsequent reperfusion phase, AMPK activity is reduced. This AMPK response to IR was observed in the heart [33]. Cardiac surgery, acute decompensated heart failure, radiocontrast dye exposure, and some therapeutics all have the likelihood of causing renal ischemia followed by reperfusion. There are currently no clinical therapies to safeguard the kidney from IR injury, and AKI significantly worsens the clinical outcomes in such situations. Our data indicate that pharmacological activation of the AMPK/autophagy axis provides beneficial cellular effects and offers a viable strategy in reducing kidney IR injury, and is thus a potential therapeutic target for future clinical intervention.

\section{Materials and Methods}

\section{Materials}

Male adult Wistar rats (Harlan Teklad) were housed and handled in accordance with VASDHS Institutional Animal Care and Use Committee and National Institutes of Health guidelines under IACUC-approved protocols. All chemicals were purchased from Sigma unless otherwise stated.

\section{IR Procedure}

Animals were pretreated with the AMPK activator metformin (Met) at $300 \mathrm{mg} / \mathrm{kg}, 24 \mathrm{~h}$ prior to $45 \mathrm{~min}$ of ischemia, or AICAR $(0.1 \mathrm{~g} / \mathrm{kg}$, i.p.) at $24 \mathrm{~h}$ prior to ischemia for $30 \mathrm{~min}$. Briefly, rats were anesthetized with Inactin $(100 \mathrm{mg} / \mathrm{kg}$ i.p.) and placed on a temperature-controlled table to maintain body temperature at $37^{\circ} \mathrm{C}$. All rats underwent unilateral nephrectomy $2-3$ weeks prior to ischemia, so compensatory growth of the remaining kidney would not be a complicating factor, and unilateral ischemia is a less traumatic procedure than bilateral ischemia in our hands. Control animals underwent a sham operation on the remaining kidney. Ischemia was accomplished by occluding the renal vascular pedicle with an atraumatic vascular clamp. Surgical incisions were sutured and reperfusion was carried out for $24 \mathrm{~h}$ after the ischemic event. Reperfusion for $24 \mathrm{~h}$ after the ischemic event leads to widespread degeneration of tubular architecture, tubular 
Fig. 1. Kidney growth and edema. Kidneyweight-to-body-weight ratio (KW:BW), and proliferating cell nuclear antigen expression increased in response to IR. Met (top) moderately decreased the growth response, but AICAR (bottom) did not. Con $=$ Control, sham. ${ }^{*} \mathrm{p}<0.05$ vs. Con; ** $\mathrm{p}<0.05$ IR vs. IR+Met or IR+AICAR. The $\mathrm{y}$-axis denotes relative densitometry units. $\mathrm{n}=4-6$ animals. Representative immunoblots are shown, and all figures were normalized with $\beta$-actin. Error bars represent \pm SEM. Statistical evaluations were performed by ANOVA with Fisher's LSD post hoc test.

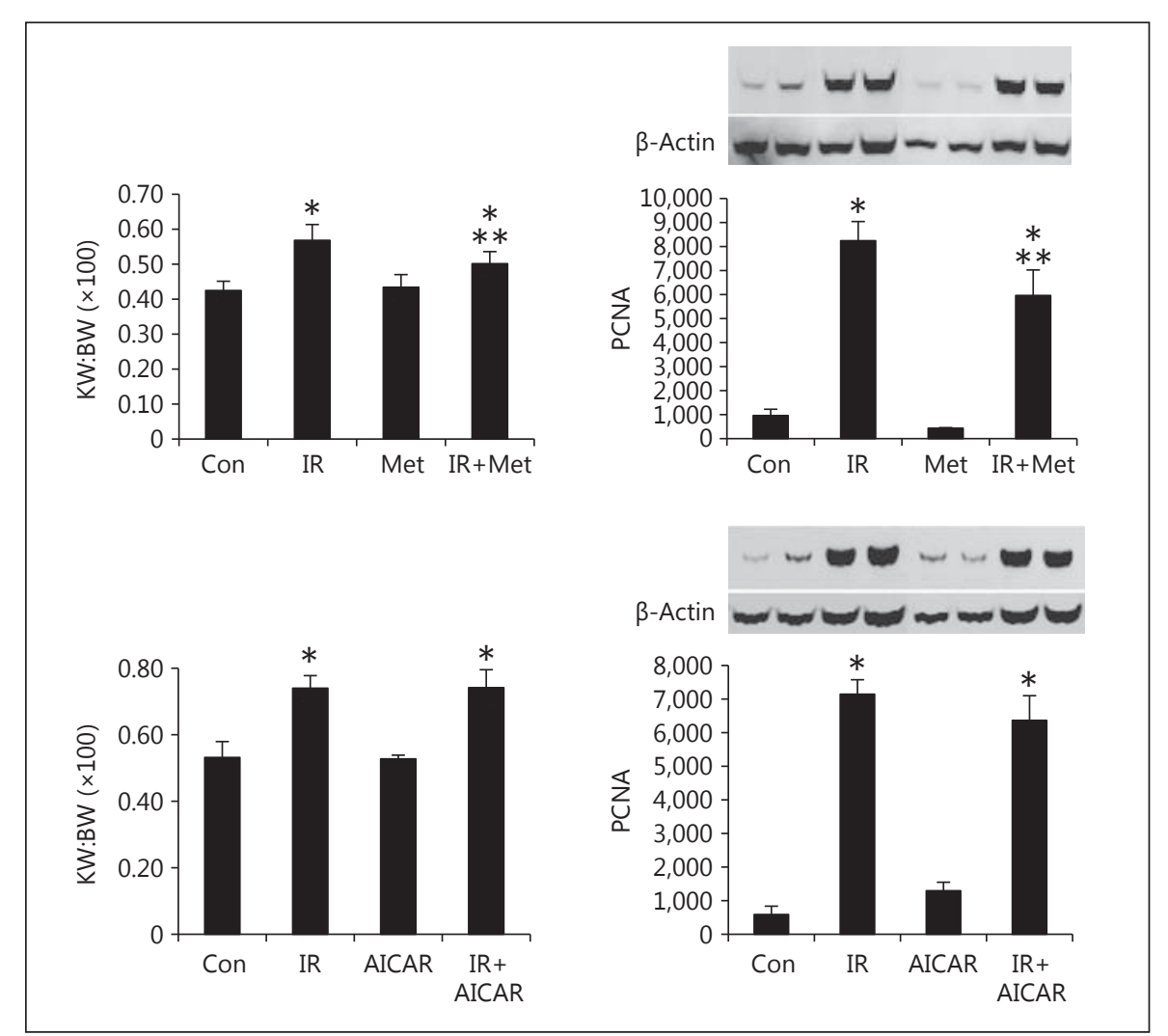

dilation, loss of brush borders, cell swelling, necrosis, and apoptosis. At $24 \mathrm{~h}$ of reperfusion, the animals were anesthetized, weighed, and the remaining kidney resected and weighed (wet weight).

\section{Western Blot Analysis}

Kidney cortices were harvested and homogenized in lysis buffer [lysis buffer: $1 \%$ triton-X 100, 0.5\% deoxycholic acid, $1 \mathrm{mM}$ EDTA, $0.1 \%$ SDS, 4 mM NaF, Complete Protease Cocktail (Roche Molecular Biochemicals, Mannheim, Germany), and $1 \mathrm{mM} \mathrm{NaVO}_{4}$ in PBS]. Lysates at $50 \mu \mathrm{g} / \mathrm{lane}$ were resolved on NuPAGE gels in MOPS buffer (Invitrogen, Carlsbad, Calif., USA). Gel proteins were transferred to nitrocellulose membranes and immunoblotted with the appropriate primary antibody, as indicated. The secondary antibody was horseradish peroxidase-conjugated (Cell Signaling Technology, Danvers, Mass., USA) for autoradiographic detection by ECL Plus (Amersham Pharmacia, Piscataway, N.J., USA), with densitometric analysis by ImageJ Software (National Institutes of Health, Bethesda, Md., USA).

\section{Histologic Analysis}

The degree of tissue injury was assessed on a semiquantitative basis by adapting a single-blind analysis of paraffin sections stained with periodic acid-Schiff reagent as detailed previously [34]. Briefly, tissue alterations were graded along an arbitrary scale for proximal tubules, distal tubules and collecting ducts evaluating hydropic degeneration, focal interruptions of brush border, necrotic or atrophic cells, and interstitial infiltration within the field.

\section{Statistical Analysis}

Results were shown as means \pm SEM. Statistics were performed by one-way ANOVA followed by Fisher's LSD post hoc testing using KaleidaGraph Software. The null hypothesis was rejected when $\mathrm{p}<0.05$.

\section{Results}

\section{Kidney Edema/Growth}

To evaluate the effects of AMPK activation in IR injury, we utilized the AMPK agonists AICAR and Met. AICAR is an AMP analog that is a cell-permeable, allosteric activator of AMPK and is currently a widely used selective agonist of AMPK for in vivo use. We also used Met in these experiments as it is inexpensive, readily available, and a widely used therapeutic. Kidney weight and kidney weight-to-body weight ratios increased with IR. Administration of Met modestly reduced this change in IR, but levels were still above control (fig. 1). Similar changes occurred for absolute kidney weight. No changes were noted with AICAR in these parameters. Proliferating cell nuclear antigen did decrease slightly but significantly in IR+Met versus IR animals, but again this change 


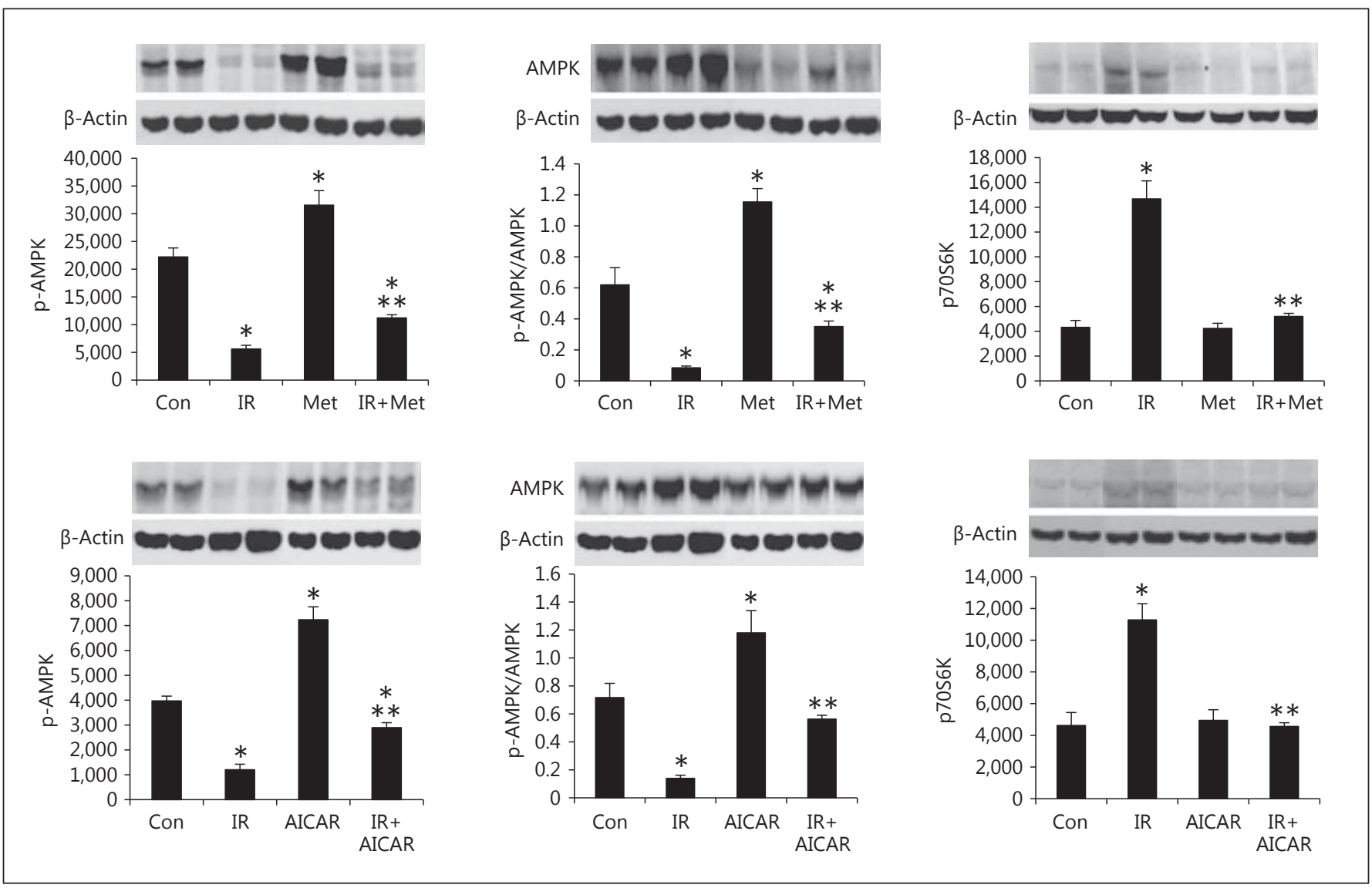

Fig. 2. $A M P K$ and $m T O R$ activities. Profile of activated p-AMPK by immunoblot. At $24 \mathrm{~h}$ of reperfusion (IR), AMPK activity is significantly below the control. Both AMPK agonists raised p-AMPK relative to the control, with IR+Met or AICAR also above IR-alone levels. A similar pattern was observed when AMPK activity was expressed as p-AMPK expression per total AMPK expression. The increase in phosphorylated p70S6K is indicative of increased mTORC1 activity. IR increases p70S6K, whereas Met or AICAR administration in IR animals normalizes these levels. These data support the counterregulation of these nutrient sensors. Con $=$ Control, sham. ${ }^{*} \mathrm{p}<0.05$ vs. Con; ${ }^{* *} \mathrm{p}<0.05$ IR vs. IR+Met or IR+AICAR. The $\mathrm{y}$-axis denotes relative densitometry units. $\mathrm{n}=$ 4-6 animals. Representative immunoblots are shown, and all figures were normalized with $\beta$-actin. Error bars represent \pm SEM. Statistical evaluations were performed by ANOVA with Fisher's LSD post hoc test. was not observed with AICAR. Thus, AMPK activators do not appear to have a major effect on the initial increase in edema and growth responses at this early, 24-hour time point.

\section{Kidney IR Affects AMPK and Mammalian Target of Rapamycin Activities}

AMPK phosphorylation at threonine 172 (T172) in the $\alpha$-subunit [35] is a key mechanism regulating AMPK activation in all tissues [36]. The IR group demonstrates a decrease of active phosphorylated AMPK (p-AMPK) and the p-AMPK/AMPK ratio, below control levels at $24 \mathrm{~h}$ after reperfusion (fig. 2). Met or AICAR, adminis- tered $24 \mathrm{~h}$ prior to ischemia, increased AMPK activities with either and in IR+Met or IR+AICAR over IR alone.

AMPK induces autophagy by direct phosphorylation of the ULK1 autophagy initiation complex (ULK1, ATG13, FIP200), and inhibition of mammalian target of rapamycin (mTOR). Whereas AMPK is activated in response to low nutrition or energy expenditure, nutrients induce mTOR activity to synthesize new proteins and fatty acids, increase metabolic activity, and inhibit autophagy. Downstream from mTORC1 is the kinase p70S6K, which correlates with mTORC1 activity. The mTORC1/ p70S6K pathway is known to suppress the autophagy process. As illustrated in figure 2, the expression of 

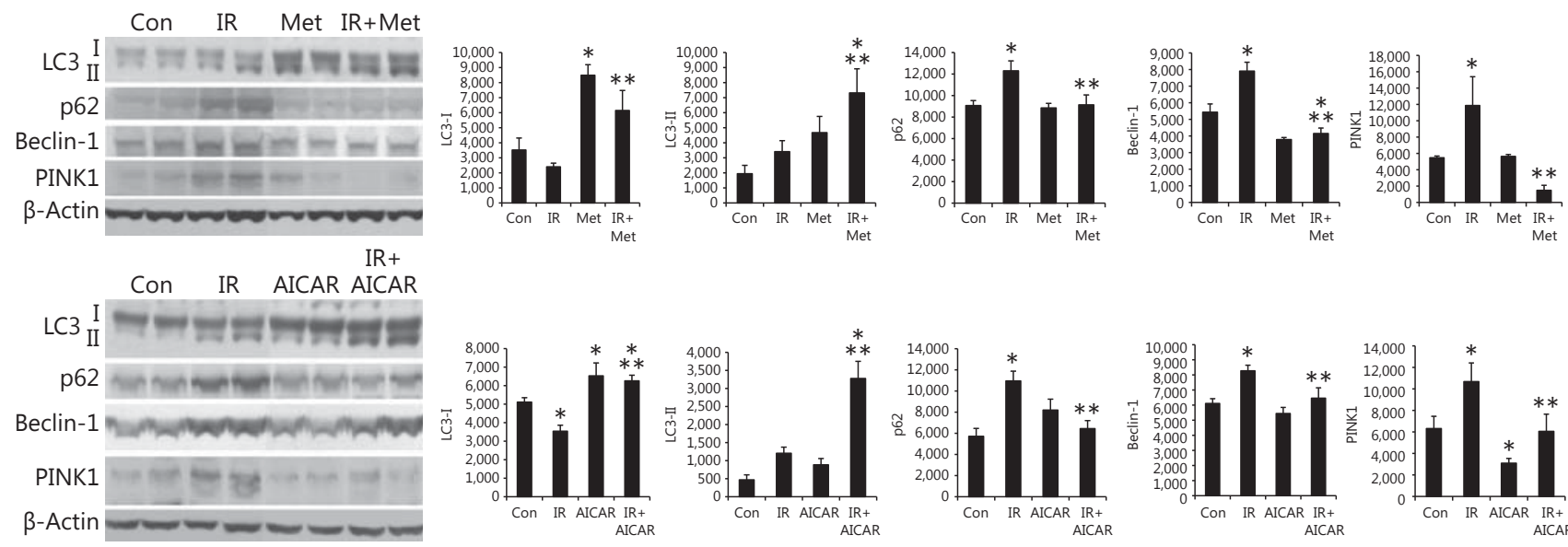

Fig. 3. Alterations in autophagy. Western blots demonstrate an increase in precursor LC3-I protein with Met (top) or AICAR (bottom) administration. The autophagy protein LC3-II trends to increase expression in IR. IR+Met or AICAR administration significantly increases this expression further. Expression of p62 increases with IR. This response to IR is normalized with administration of Met or AICAR. Beclin-1, which can promote autophagy independent of AMPK, increases with IR. PINK1, a selective mi-

p70S6K significantly increased in the IR group (fig. 2) while its expression was prevented by AICAR and Met, suggesting that the induction of AMPK activity can lower the mTORC1/p70S6K response from inhibiting the autophagy process after an IR insult.

\section{Kidney IR Affects Autophagy}

To evaluate autophagy, we utilized immunoblotting of microtubule-associated protein light chain 3 (LC3) and p62/SQSTM1 (p62) expressions in rat kidney cortices. Upon induction of autophagy, LC3-I becomes incorporated into the autophagosome membrane through lipidation with phosphatidylethanolamine to become LC3II. LC3-I and LC3-II are established indicators of autophagy [37] and migrate as a double band of upper cytosolic LC3-I and lower autophagosome bound LC3-II (fig. 3). As illustrated in figure 3, a decrease in LC3-I and corresponding increase in LC3-II were observed in response to IR. Therefore, this increase in autophagy along with decreased AMPK and increased MTORC1 activities imply an induction in response to a stress signal rather than nutritional status. However, the administration of AICAR or Met (AMPK agonist) in control rats signifi- tophagic protein, also increases with IR. Both of these proteins are normalized with administration of Met or AICAR in IR animals. Con $=$ Control, sham. ${ }^{*} \mathrm{p}<0.05$ vs. Con; ${ }^{* *} \mathrm{p}<0.05$ IR vs. IR+Met or IR+AICAR. The $\mathrm{y}$-axis denotes relative densitometry units. $\mathrm{n}=$ 4-6 animals. Representative immunoblots are shown. All figures were normalized with $\beta$-actin; representative $\beta$-actin bands are shown. Error bars represent \pm SEM. Statistical evaluations were performed by ANOVA with Fisher's LSD post hoc test. cantly increased the LC3-I level. This LC3-I increase was also observed in the AICAR- and Met-treated IR rats. The capacity of Met and AICAR to increase autophagy is consistent with autophagy being downstream from AMPK.

Increases in LC3-I would be permissive for a rapid and increased response, and this we observe with LC3-II in IR + Met or IR+AICAR (fig. 3). Agonist activation of AMPK activity does increase cytosolic LC3-I, but does not translate into a marked LC3-II response until the IR stimulus. This implies the agonists prime the system to allow for a rapid increase in autophagy that only occurs in response to appropriate stimulation.

The adapter protein p62/SQSTM1 (p62) acts as a signaling hub through its capacity to recruit proteins and signaling molecules. There are several binding domains on p62, including an ubiquitin-associated binding domain and an LC3 interacting region. In this way p62 scavenges cytosolic proteins tagged with ubiquitin for degradation and binds LC3 to tether these proteins into the forming autophagosome for degradation. Here we observed a significant increase in p62 expression at $24 \mathrm{~h}$ after IR with administration of Met or AICAR normalizing 


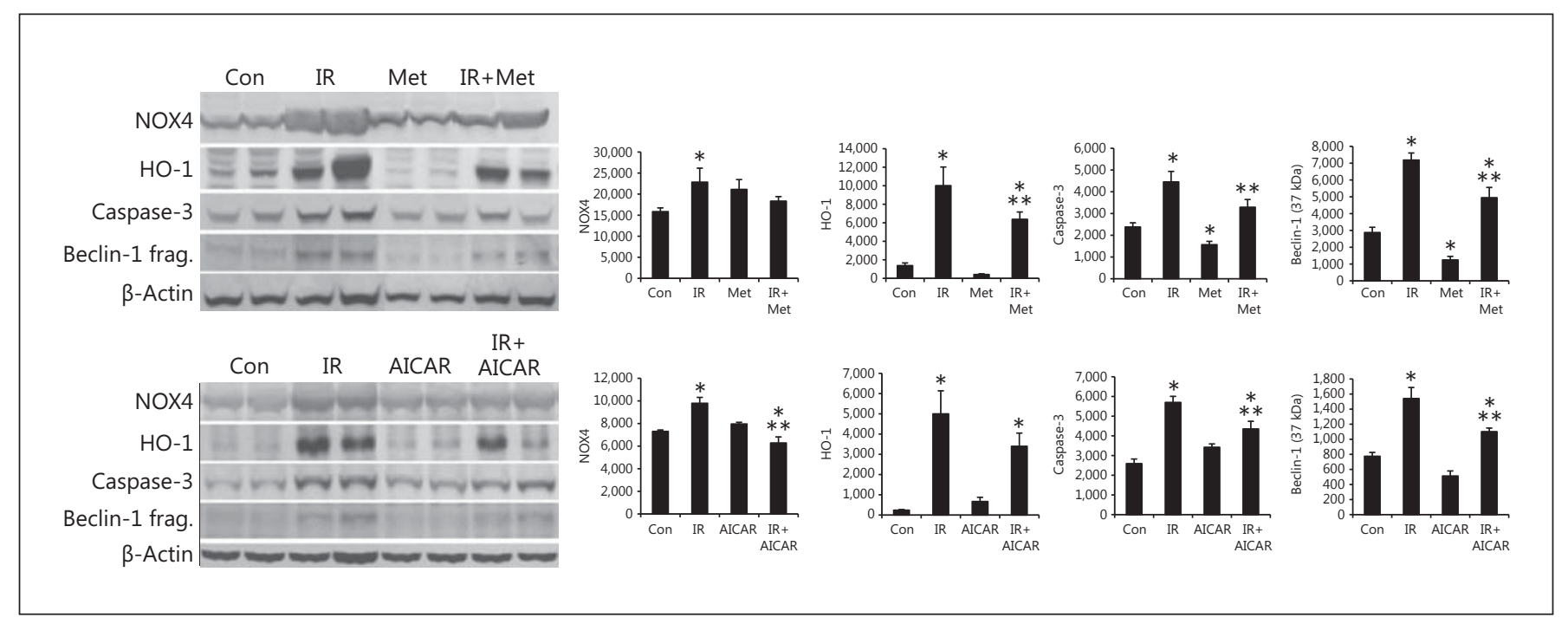

Fig. 4. Indicators of cellular stress. NOX4 expression increases with IR, but is not different from Con with IR+Met (top) or IR+AICAR (bottom) administration. HO-1, a redox stress-related protein, correlates with NOX4 induction in IR and also decreases with IR+Met or AICAR. Apoptosis is well described in IR, and cleaved caspase- 3 and Beclin-1 fragment induction by kidney IR are also significantly increased with IR. These expressions are attenuated with AMPK agonist administration, IR+Met or
IR+AICAR. Con $=$ Control, sham. ${ }^{*} \mathrm{p}<0.05$ vs. Con; ${ }^{* *} \mathrm{p}<0.05$ IR vs. IR+Met or IR+AICAR. The y-axis denotes relative densitometry units. $n=4-6$ animals. Representative immunoblots are shown. All figures were normalized with $\beta$-actin; representative $\beta$-actin bands are shown. Error bars represent \pm SEM. Statistical evaluations were performed by ANOVA with Fisher's LSD post hoc test. these levels in the kidney cortex samples (fig. 3). This normalization would correlate with the increased autophagy observed by LC3-II expression with AMPK agonists and indicate that general autophagy under IR is insufficient to clear p62 aggregates.

To further delineate the effect of AMPK activation in the autophagy process after IR we also measured Beclin-1, which is the mammalian homolog of yeast Atg6, a key protein in the initiation of autophagosome formation. Contrary to nutritional sensor regulation of autophagy, ROS-dependent Beclin-1-mediated autophagy has been shown to be detrimental in the IR model in the heart [30, 33]. As illustrated in figure 3, Beclin-1 increased in IR animals (fig. 3). Again, this response was normalized with administration of the AMPK agonists Met or AICAR.

Finally, we investigated PTEN-induced putative kinase-1 (PINK1). The rapidly degraded mitochondrial protein, PINK1, is stabilized by mitochondrial depolarization [38], which begins sequential steps in the selective autophagic degradation of the mitochondria, or mitophagy [39-41]. Mitophagy is an efficient mechanism for the cell to maintain quality control over aged or damaged mitochondria to avoid cell death [42-44]. As observed, the significant induction of PINK1 in IR rats was normalized with the addition of AMPK agonists (fig. 3).

\section{AMPK Activation Decreases IR-Related Cellular Stress}

It is well established that IR increases cellular stress. Here we evaluate four cellular markers to verify this occurrence in our model and to determine the effects of AMPK agonists on these markers.

Extensive tubular apoptosis and necrosis and a compromised interstitium attributed to oxidative stress resulting in architectural degradation are the hallmarks of this disease model. We evaluated NOX4, an oxidase that emits hydrogen peroxide $\left(\mathrm{H}_{2} \mathrm{O}_{2}\right)$, as a potential contributor of oxidative stress. NOX4 was upregulated in the IR group, but this induction was significantly attenuated in the IR+AICAR group, with a similar profile trend in IR+Met (fig. 4). Heme oxygenase-1 (HO-1) is another indicator of cellular oxidative stress induced in IR. Induction of HO-1 confers protection against tissue injury caused by diverse stressors including ischemia and oxidative stress. p62 aggregates stabilize the transcription factor Nrf-2, which upregulates a panel of cellular antioxidants, including HO-1. Here, the significant increase in $\mathrm{HO}-1$ level in the IR group 


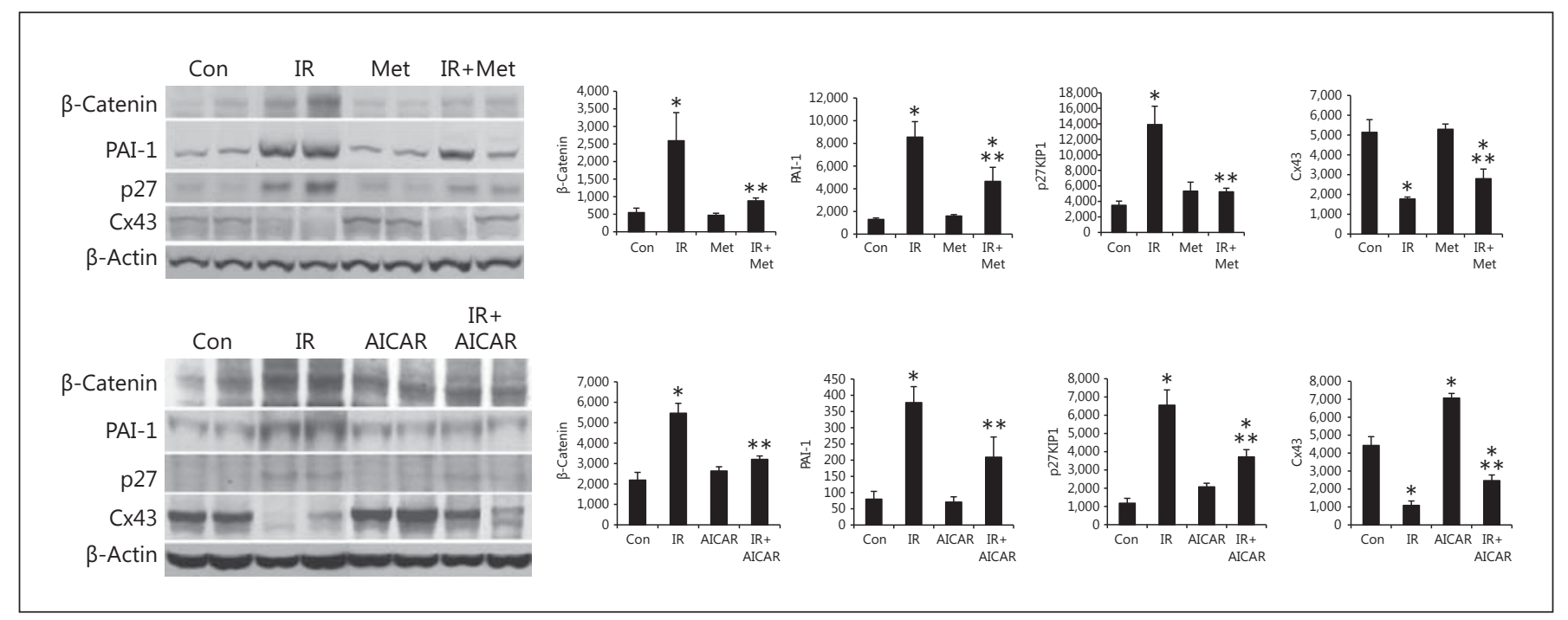

Fig. 5. Profibrotic markers. Early indicators of fibrosis, $\beta$-catenin and PAI-1, are increased by IR at this early time point prior to histologic detection of fibrosis. The cyclin-dependent kinase inhibitor, p27, is induced in a number of kidney pathologies and is increased with IR. Cx43 decreases with IR. All of these changes are attenuated with administration of AMPK agonists. Con = Control, sham. ${ }^{*} \mathrm{p}<0.05$ vs. Con; ${ }^{* *} \mathrm{p}<0.05$ IR vs. IR+Met or IR+AICAR. The $\mathrm{y}$-axis denotes relative densitometry units. $\mathrm{n}=4-6$ animals. Representative immunoblots are shown. All figures were normalized with $\beta$-actin; representative $\beta$-actin bands are shown. Error bars represent \pm SEM. Statistical evaluations were performed by ANOVA with Fisher's LSD post hoc test. was prevented by Met administration (IR+Met; fig. 4). However, the large variation of HO-1 expressions in IR animals precluded significance versus IR+AICAR.

While apoptosis is a central tenet in the extensive loss of tubular epithelial cells after kidney IR injury, we decided to measure cleaved caspase-3. As illustrated, cleaved caspase- 3 was increased in the IR group. This IR induction in cleaved caspase- 3 was abated in IR+Met- or IR+AICAR-treated animals (fig. 4). Here we observed an increase in Beclin-1 fragments with IR. The decrease in caspase- 3 in IR+AMPK agonists is consistent with the reduction of these proapoptotic fragments.

\section{AMPK Activation Attenuates IR Increases in Profibrotic Markers}

AKI can lead to long-term effects in the kidney, such as tissue scarring. Wnt $/ \beta$-catenin is required for TGF- $\beta$ mediated fibrosis [45] and may be a common factor in this progression [46, 47]. Plasminogen activator inhibitor-1 (PAI-1) inhibits plasminogen activation and thus fibrinolysis. Upregulation and suppression of fibrolytic activity by PAI- 1 appears to play a significant role in the progression to fibrosis. The cyclin kinase-dependent inhibitor p27KIP1 is induced in a number of kidney pathologies, and has been shown to be a required intermedi- ary between the induction of TGF- $\beta 1$ and generation of collagen and fibronectin in a model of type 1 diabetes mellitus $[48,49]$. Therefore, as illustrated in figure 5 , the upregulation of $\beta$-catenin, PAI-1, and p27KIP1 expressions observed in the IR group are indicative of markers preceding fibrosis (fig. 5). Induction of AMPK activity by Met or AICAR reduced all three of these protein expressions in IR animals.

Connexin $43(\mathrm{Cx} 43)$ is a gap junction protein differentially distributed throughout the different segments of the kidney [50] and maintains a protective role in cell homeostasis and defense. Downregulation of Cx43 and cell-cell communication in IR is thought to afford protection from inflammation and necrosis by limiting the spread of signaling molecules associated with extensive IR damage. In our model, the $\mathrm{Cx} 43$ was significantly decreased in the IR group while its downregulation was observed to a lesser extent in IR with Met or AICAR administration. This could imply a reduced cellular damage and increased cellcell communication (fig. 5).

\section{IR Impacts Kidney Tubules and the Interstitium: \\ Histologic Analysis}

Since IR injury leads to tubular alteration, the histological assessment of IR damage to the cortex is shown in 
Fig. 6. Histopathological alterations. Representative kidney cortex sections stained with periodic acid-Schiff reagent for the untreated control (a), control+AICAR (b), $\operatorname{IR}(\mathbf{c}, \mathbf{d})$, and IR+AICAR (e, f) groups. Histological changes (injury scores) found in the tubules ( $\mathbf{g})$ and interstitium (h). The arrowhead indicates proximal tubular epithelial cell desquamation. The arrow illustrates the denudation of the proximal tubular basement membrane. The loss of brush border in proximal tubular cells is shown by the dotted arrow, while the asterisk illustrates the presence of cast in collecting duct. Each paraffin section was scanned at $\times 400$ magnification with a microscope equipped with an eyepiece containing a $1-\mathrm{cm}^{2}$ grid for reference. Twenty square fields, each covering an area of $0.084 \mathrm{~mm}^{2}$, were evaluated for each section. $\mathrm{PT}=$ Proximal tubules; $\mathrm{G}=$ glomerulus; $\mathrm{NT}=$ necrotic tubules. ${ }^{*} \mathrm{p}<0.05$ vs. Con.
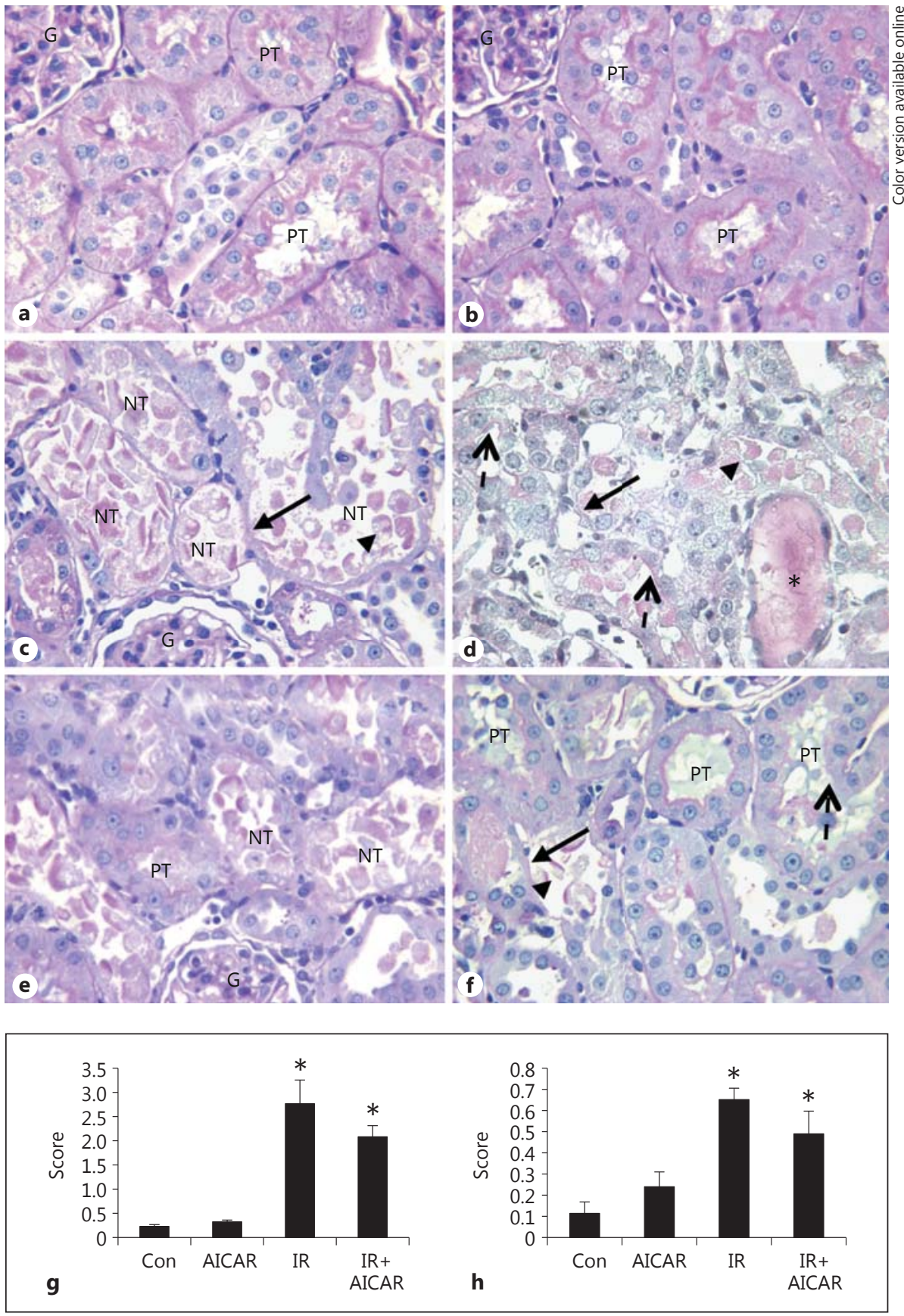

figure 6. The kidney displayed the usual features related to this well-known model of kidney IR. As illustrated, the degree of severity of tubular damages was significantly high in the IR group compared to the control group (fig. 6g). Tubular alterations were mostly located in proximal tubules and were characterized by losses of brush border (dotted arrow in fig. 6d), cytoplasmic edema, pyknotic nuclei, and cell necrosis. This severe tubular necro- sis was associated with epithelial cell desquamation (fig. 6, arrowhead) and denudation of tubular basement membrane (fig. 6, arrow). In the lumen of collecting ducts, periodic acid-Schiff-positive staining illustrated the presence of casts which is a sign of an onset of proteinuria (fig. 6d, asterisk).

Although tubular damage was still apparent, AICAR treatment reduced the extent and severity of these lesions 
into the proximal tubules by comparison with the IR group. In the interstitium, a significant increase of interstitial cell density was observed in the IR group. Again, this change was attenuated by AICAR treatment (fig. 6f). No morphological lesions were observed in glomeruli at this time point.

\section{Discussion}

The early response initiated by IR is characterized by a rapid cascade of events prompting cellular damage that often overwhelms the responsive cellular protective mechanisms, thus leading to cell death and organ dysfunction. Our current results highlight the effects that IR has on downregulation of renoprotective AMPK and autophagy and consequent cellular changes.

Autophagy is a homeostatic mechanism of cellular quality control and a beneficial adaptive response mechanism to cellular stress or pathologic insult. However, there is still some controversy about the role of autophagy in the context of AKI. In proximal tubule cells subject to oxidative stress via hydrogen peroxide or hypoxia, an increase in autophagy accompanied an increase in cell death. Blocking autophagy with lysosomal protease inhibitors suppressed cell death in response to these stressors [51].

Similarly, cisplatin treatment of proximal tubule cells increased autophagy and cell death, with administration of selective autophagy inhibitors or Beclin-1 siRNA to block autophagy, again decreasing cell death in this model of AKI [52]. The authors surmised that tubule cells could be protected from AKI-induced cell death by suppressing autophagy, and thus a rapid and excessive autophagy in these models may lead to cell death. Conversely, in cisplatin-treated renal tubule epithelial cells, autophagic blockade with the inhibitor 3-methyladenine led to increased apoptosis [53]. Furthermore, suppression of autophagy induction in the IR model by use of chloroquine or 3-methyladenine in cells in culture or in vivo resulted in worsening cellular damage and renal function, indicating beneficial effects of autophagy in this model [54]. Mice conditionally deficient in Atg5 or Atg7 in proximal tubule cells in response to IR or cisplatin, respectively, also demonstrated an increase in apoptosis and decrease in kidney function relative to wild-type mice, implying autophagy is protective in these AKI models $[55,56]$.

The apparent contradiction in these experiments regarding the role of autophagy may simply be attributed to differences in cell lines, treatments, or magnitude of the insult resulting in an excessive and lethal autophagic response. Alternatively, blocking autophagy by either pharmacologic or genetic means will suppress the necessary homeostatic functions of autophagy, placing these cells at a disadvantage by sensitizing them and compounding the injury. Even in the elegantly performed investigations utilizing genetic models, for example, an increase in p62 is observed in the otherwise untreated knockout animals.

An increase in p62 implies insufficient autophagic flux to remove and degrade p62 and its cargo proteins [57]. This led us to take a different approach by inducing rather than blocking autophagy by agonist induction of the natural upstream regulator of autophagy, AMPK. This is of particular interest as we find AMPK exhibits decreased activity in the kidneys of IR animals and these experiments evaluate the effects of reinstating AMPK activity. The advantage this approach has over mTOR inhibition to induce autophagy is the recent observation that inhibition of mTOR in podocytes suppresses new lysosome formation [58]. The autophagic process therefore becomes uncoupled as witnessed by an accumulation of autophagosomes unable to degrade their cargo. Here we induce autophagy with AMPK agonists to determine if reinstating AMPK activity to normal levels as a mode to increase autophagy beyond that of IR alone exacerbates injury or has beneficial cellular effects. If excess autophagy is responsible for increased cell death, this approach should increase injury and the decrease in AMPK as a regulator of autophagy would be considered protective.

Although we do observe an increase in the autophagy marker LC3-II, our observation of increased p62 in IR kidneys indicates that autophagy, or at least general macroautophagy, may be less than adequate under these conditions to sequester and clear cellular cargo. The inability to dispose of toxic cellular aggregates increases cell stress and decreases longevity. Induction of the AMPK/autophagy axis did improve the cell markers evaluated, supporting autophagy as a beneficial protective mechanism in the IR model of AKI. How the AMPK-autophagy axis affects these stress markers requires further examination. The effects from a chronic imbalance in catabolic and anabolic mechanisms in regard to autophagy have been reviewed [59].

Stratifying the ischemic and reperfusion phases into AMPK- and ROS-induced autophagy components, respectively, may further explain the paradoxical effects of earlier experiments. In the heart, ischemia-induced autophagy mediated by AMPK garnered beneficial effects; however, in the reperfusion phase, Beclin-1-dependent, 
AMPK-independent autophagy was deemed maladaptive due to worsened myocardial injury $[32,33]$. Thus, the mode of autophagy induction may yield different results. As shown in the heart [33], and here in the kidney, the nutritional sensor AMPK decreases its activity during the nutrient-rich reperfusion phase. Reperfusion then exhibits increasing autophagy that is independent of nutrients and AMPK, but dependent on ROS [33].

Whereas AMPK is associated with beneficial autophagy, reperfusion induction of ROS-dependent Beclin-1 mediated forms may reflect autophagy associated with detrimental effects [30,33]. Detrimental Beclin-1-associated autophagy is also described in the kidney in cisplatin nephrotoxicity [60]. High ROS would be suggestive of mitochondrial damage and the Beclin-1 autophagy may constitute a protective response to rid the cell of damaged mitochondria. Here we further support mitophagy by the observation of an increase in Beclin-1, but importantly also an increase in the selective mitophagy adapter protein, PINK1, in our IR model.

Mitochondrial depolarization stabilizes PINK1 [38], which then recruits the cytosolic E3 ubiquitin ligase component protein, Parkin, to ubiquitinate the mitochondria for degradation via mitophagy [39-41]. The stable expression of this mitophagy-specific protein may function as a 'quality control' mechanism to safeguard cells from aged or dysfunctional mitochondria and associated oxidative stress and apoptosis [42-44]. However, extensive mitophagy without the protective effects and biogenesis of new mitochondria afforded by AMPK, via PGC-1a for example, could leave a cell devoid of sufficient mitochondria for survival while still accumulating damaged proteins and organelles. Such a scenario could result in caspase-independent programmed cell death.

The normalizations in p62, Beclin-1, and PINK1 expressions in IR+Met or IR+AICAR animals, along with the increase in LC3-II, would be supportive of primary homeostatic autophagy provided by AMPK activation. Additionally, a shift from general autophagy to selective mitophagy may be a transient response to failed mitochondria, but the magnitude or duration of this shift could leave the cell lacking in the homeostatic and protective effects of canonical autophagy. Further characterization of these autophagic programs may be required in models of AKI.

Tubular injury and death are characteristic pathologic consequences of IR. Formation of extracellular matrix is a healing and protective response to injury. It can turn harmful if it becomes chronic and independent from the initial stimulus leading to fibrosis, scarring, and disrup- tion of the tissue architecture and function. Some areas of the IR-damaged kidney progress to fibrosis along with other lesions, which is a poor prognostic factor. Dysregulation of the canonical Wnt/ $\beta$-catenin-signaling pathways underlie the pathogenesis of renal fibrosis [47]. TGF- $\beta$ induces $\mathrm{Wnt} / \beta$-catenin [45] to upregulate PAI-1 expression in kidney tubule cells [61], whereas autophagy decreases Wnt activity by degrading its signaling protein Dishevelled $[62,63]$. Further, AMPK suppresses TGF- $\beta$ induced secretion of collagen I and IV and fibronectin [4], which may also attenuate Wnt signaling downstream from TGF- $\beta$.

Although $24 \mathrm{~h}$ after ischemia is too early for fibrosis, we have observed an increase in both $\beta$-catenin and PAI1 in response to IR, whereas induction of AMPK/autophagy attenuates that effect in IR animals. The cyclin-dependent kinase inhibitor p 27 was required in TGF- $\beta 1$ induction of collagen and fibronectin in diabetes models $[48,49]$, and here demonstrates the same response pattern as $\beta$-catenin and PAI-1. Gap junction channels such as $\mathrm{Cx} 43$ provide low resistance pathways to allow current, ions, and small molecules $(<1,000 \mathrm{Da})$ to pass between neighboring cells. As such they contribute to metabolic homeostasis and synchronization of the cellular environment through intercellular movement of metabolites, ions, second messengers, and electrical cell-to-cell coupling.

Induction of $\mathrm{p} 27$ along with PAI- 1 and the downregulation of $\mathrm{Cx} 43$, as observed here, are indicative of premature or accelerated senescence as we previously reported for diabetes [64]. Although $24 \mathrm{~h}$ is too early a time point to verify premature senescence, transition to a senescent phenotype would prove detrimental as it leads to increased cellular oxidant production [65], increased oxidation of proteins $[66,67]$, an increased inflammatory response [68], and increases in TGF- $\beta$ and fibrosis [69]. Aberrant expressions of these predictive markers of senescence are attenuated with Met or AICAR administration to IR animals.

A recent study from Lempiäinen et al. [70] evaluated kidney physiology and the inflammatory response in kidney IR with the administration of AICAR. In this study, which was also evaluated at $24 \mathrm{~h}$ of reperfusion, serum creatinine and acute tubule necrosis were significantly reduced at $500 \mathrm{mg} / \mathrm{kg}$ AICAR, but not at the lower concentrations. We also did not observe significant changes in kidney histology at this early time point, but a shift in the molecular markers was apparent even at a AICAR dose five times lower that utilized in our studies. The data from Lempiäinen et al. [70], along with our current data, sug- 
gest that AMPK/autophagy may have a lesser role in arresting the acute injury, and instead a larger role in cellular adaptation to the injury.

$\mathrm{AKI}$ is a major health issue with increasing incidence worldwide and is frequently seen among patients with cardiovascular conditions [71]. Our data indicate that pharmacological activation of AMPK with Met or AICAR provides beneficial cellular effects and offers a viable strategy for reducing progressive components of injury in kidney IR.

\section{Acknowledgements}

We would like to thank Mr. Ser Khang for his time and surgical skill in performing the IR operations on the animals. This work is supported by NIH grants DK094352, DK083142, DK02920, DK56248, and DK28602, the UAB-UCSD O'Brien Center for Acute Kidney Injury Research P30DK079337, and the Department of Veterans Affairs, Veterans Health Administration, Office of Research and Development.

\section{Disclosure Statement}

All authors declare no competing interests.

\section{References}

1 Carling D, Mayer FV, Sanders MJ, Gamblin SJ: AMP-activated protein kinase: nature's energy sensor. Nat Chem Biol 2011;7:512518.

-2 Steinberg GR, Kemp BE: AMPK in health and disease. Physiol Rev 2009;89:1025-1078.

-3 Xie Z, Zhang J, Wu J, Viollet B, Zou MH: Upregulation of mitochondrial uncoupling protein- 2 by the AMP-activated protein kinase in endothelial cells attenuates oxidative stress in diabetes. Diabetes 2008;57:32223230.

4 Mishra R, Cool BL, Laderoute KR, Foretz M, Viollet B, Simonson MS: AMP-activated protein kinase inhibits transforming growth factor-beta-induced Smad3-dependent transcription and myofibroblast transdifferentiation. J Biol Chem 2008;283:10461-10469.

5 Eid AA, Ford BM, Block K, Kasinath BS, Gorin Y, Ghosh-Choudhury G, Barnes JL, Abboud HE: AMP-activated protein kinase (AMPK) negatively regulates Nox4-dependent activation of p53 and epithelial cell apoptosis in diabetes. J Biol Chem 2010;285: 37503-37512.

6 Harhaji-Trajkovic L, Vilimanovich U, Kravic-Stevovic T, Bumbasirevic V, Trajkovic V: AMPK-mediated autophagy inhibits apoptosis in cisplatin-treated tumour cells. J Cell Mol Med 2009;13:3644-3654

-7 Kim JE, Ahn MW, Baek SH, Lee IK, Kim YW, Kim JY, Dan JM, Park SY: AMPK activator, AICAR, inhibits palmitate-induced apoptosis in osteoblast. Bone 2008;43:394404.

8 Nelyudova A, Aksenov N, Pospelov V, Pospelova T: By blocking apoptosis, Bcl-2 in p38-dependent manner promotes cell cycle arrest and accelerated senescence after DNA damage and serum withdrawal. Cell Cycle 2007;6:2171-2177
-9 Rebbaa A, Zheng X, Chou PM, Mirkin BL: Caspase inhibition switches doxorubicin-induced apoptosis to senescence. Oncogene 2003;22:2805-2811.

10 Short JD, Houston KD, Dere R, Cai SL, Kim J, Johnson CL, Broaddus RR, Shen J, Miyamoto S, Tamanoi F, Kwiatkowski D, Mills GB, Walker CL: AMP-activated protein kinase signaling results in cytoplasmic sequestration of p27. Cancer Res 2008;68:64966506.

11 Young AR, Narita M, Ferreira M, Kirschner K, Sadaie M, Darot JF, Tavare S, Arakawa S, Shimizu S, Watt FM: Autophagy mediates the mitotic senescence transition. Genes Dev 2009;23:798-803.

12 Patschan S, Chen J, Polotskaia A, Mendelev N, Cheng J, Patschan D, Goligorsky MS: Lipid mediators of autophagy in stress-induced premature senescence of endothelial cells. Am J Physiol Heart Circ Physiol 2008; 294:H1119-H1129.

13 Andrews ZB, Liu ZW, Wallingford N, Erion DM, Borok E, Friedman JM, Tschop MH, Shanabrough M, Cline G, Shulman GI, Coppola A, Gao XB, Horvath TL, Diano S: UCP2 mediates ghrelin's action on NPY/AgRP neurons by lowering free radicals. Nature 2008;454:846-851.

14 Canto C, Auwerx J: AMP-activated protein kinase and its downstream transcriptional pathways. Cell Mol Life Sci 2010;67:34073423.

15 Gaskin FS, Kamada K, Yusof M, Durante W, Gross G, Korthuis RJ: AICAR preconditioning prevents postischemic leukocyte rolling and adhesion: role of K(ATP) channels and heme oxygenase. Microcirculation 2009;16: 167-176.

16 Lim A, Park SH, Sohn JW, Jeon JH, Park JH, Song DK, Lee SH, Ho WK: Glucose deprivation regulates KATP channel trafficking via AMP-activated protein kinase in pancreatic beta-cells. Diabetes 2009;58:2813-2819.
17 Reznick RM, Shulman GI: The role of AMPactivated protein kinase in mitochondrial biogenesis. J Physiol 2006;574:33-39.

18 Suwa M, Nakano H, Kumagai S: Effects of chronic AICAR treatment on fiber composition, enzyme activity, UCP3, and PGC-1 in rat muscles. J Appl Physiol 2003;95:960-968.

19 Zong H, Ren JM, Young LH, Pypaert M, Mu J, Birnbaum MJ, Shulman GI: AMP kinase is required for mitochondrial biogenesis in skeletal muscle in response to chronic energy deprivation. Proc Natl Acad Sci USA 2002; 99:15983-15987.

20 Pallet N, Anglicheau D: Autophagy: a protective mechanism against nephrotoxicantinduced renal injury. Kidney Int 2009;75 118-119, author reply 119 .

21 Gustafsson AB, Gottlieb RA: Recycle or die: the role of autophagy in cardioprotection. J Mol Cell Cardiol 2008;44:654-661.

22 Park HK, Chu K, Jung KH, Lee ST, Bahn JJ, Kim M, Lee SK, Roh JK: Autophagy is involved in the ischemic preconditioning. Neurosci Lett 2009;451:16-19.

23 Kundu M, Thompson CB: Autophagy: basic principles and relevance to disease. Annu Rev Pathol 2008;3:427-455.

24 Mizushima N, Levine B, Cuervo AM, Klionsky DJ: Autophagy fights disease through cellular self-digestion. Nature 2008;451: 1069-1075.

25 Levine B, Kroemer G: Autophagy in aging, disease and death: the true identity of a cell death impostor. Cell Death Differ 2009;16: $1-2$

26 Williams A, Jahreiss L, Sarkar S, Saiki S, Menzies FM, Ravikumar B, Rubinsztein DC: Aggregate-prone proteins are cleared from the cytosol by autophagy: therapeutic implications. Curr Top Dev Biol 2006;76:89-101. 
27 Nakai A, Yamaguchi O, Takeda T, Higuchi Y, Hikoso S, Taniike M, Omiya S, Mizote I, Matsumura Y, Asahi M, Nishida K, Hori M, Mizushima N, Otsu K: The role of autophagy in cardiomyocytes in the basal state and in response to hemodynamic stress. Nat Med 2007;13:619-624.

28 Mathew R, Kongara S, Beaudoin B, Karp CM, Bray K, Degenhardt K, Chen G, Jin S, White E: Autophagy suppresses tumor progression by limiting chromosomal instability. Genes Dev 2007;21:1367-1381.

-29 Meley D, Bauvy C, Houben-Weerts JH, Dubbelhuis PF, Helmond MT, Codogno P, Meijer AJ: AMP-activated protein kinase and the regulation of autophagic proteolysis. J Biol Chem 2006;281:34870-34879.

- 30 Takagi H, Matsui Y, Hirotani S, Sakoda H, Asano T, Sadoshima J: AMPK mediates autophagy during myocardial ischemia in vivo. Autophagy 2007;3:405-407.

-31 Nishida Y, Arakawa S, Fujitani K, Yamaguchi H, Mizuta T, Kanaseki T, Komatsu M, Otsu K, Tsujimoto Y, Shimizu S: Discovery of Atg5/Atg7-independent alternative macroautophagy. Nature 2009;461:654-658.

-32 Matsui Y, Kyoi S, Takagi H, Hsu CP, Hariharan N, Ago T, Vatner SF, Sadoshima J: Molecular mechanisms and physiological significance of autophagy during myocardial ischemia and reperfusion. Autophagy 2008; 4:409-415.

- 33 Matsui Y, Takagi H, Qu X, Abdellatif M, Sakoda H, Asano T, Levine B, Sadoshima J: Distinct roles of autophagy in the heart during ischemia and reperfusion: roles of AMPactivated protein kinase and Beclin 1 in mediating autophagy. Circ Res 2007;100:914922.

- 34 Morin NJ, Laurent G, Nonclercq D, Toubeau G, Heuson-Stiennon JA, Bergeron MG, Beauchamp D: Epidermal growth factor accelerates renal tissue repair in a model of gentamicin nephrotoxicity in rats. Am J Physiol 1992;263:F806-F811.

- 35 Hawley SA, Davison M, Woods A, Davies SP, Beri RK, Carling D, Hardie DG: Characterization of the AMP-activated protein kinase kinase from rat liver and identification of threonine 172 as the major site at which it phosphorylates AMP-activated protein kinase. J Biol Chem 1996;271:27879-27887.

-36 Hardie DG: Sensing of energy and nutrients by AMP-activated protein kinase. Am J Clin Nutr 2011;93:S891-S896.

- 37 Kabeya Y, Mizushima N, Ueno T, Yamamoto A, Kirisako T, Noda T, Kominami E, Ohsumi Y, Yoshimori T: LC3, a mammalian homologue of yeast Apg8p, is localized in autophagosome membranes after processing. EMBO J 2000;19:5720-5728.
8 Matsuda N, Sato S, Shiba K, Okatsu K, Saisho K, Gautier CA, Sou YS, Saiki S, Kawajiri S, Sato F, Kimura M, Komatsu M, Hattori N, Tanaka K: PINK1 stabilized by mitochondrial depolarization recruits Parkin to damaged mitochondria and activates latent Parkin for mitophagy. J Cell Biol 2010;189:211-221.

9 Geisler S, Holmstrom KM, Skujat D, Fiesel FC, Rothfuss OC, Kahle PJ, Springer W: PINK1/Parkin-mediated mitophagy is dependent on VDAC1 and p62/SQSTM1. Nat Cell Biol 2010;12:119-131.

40 Vives-Bauza C, Zhou C, Huang Y, Cui M, de Vries RL, Kim J, May J, Tocilescu MA, Liu W, Ko HS, Magrane J, Moore DJ, Dawson VL, Grailhe R, Dawson TM, Li C, Tieu K, Przedborski S: PINK1-dependent recruitment of Parkin to mitochondria in mitophagy. Proc Natl Acad Sci USA 2010;107:378383.

41 Youle RJ, Narendra DP: Mechanisms of mitophagy. Nat Rev Mol Cell Biol 2011;12:914.

42 Narendra DP, Youle RJ: Targeting mitochondrial dysfunction: role for PINK1 and Parkin in mitochondrial quality control. Antioxid Redox Signal 2011;14:1929-1938.

43 Novak I: Mitophagy: a complex mechanism of mitochondrial removal. Antioxid Redox Signal 2012;17:794-802.

44 Koh H, Chung J: PINK1 as a molecular checkpoint in the maintenance of mitochondrial function and integrity. Mol Cells 2012; 34:7-13.

45 Akhmetshina A, Palumbo K, Dees C, Bergmann C, Venalis P, Zerr P, Horn A, Kireva T, Beyer C, Zwerina J, Schneider H, Sadowski A, Riener MO, MacDougald OA, Distler O, Schett G, Distler JH: Activation of canonical Wnt signalling is required for TGF- $\beta$ mediated fibrosis. Nat Commun 2012;3:735.

46 Surendran K, Schiavi S, Hruska KA: Wntdependent beta-catenin signaling is activated after unilateral ureteral obstruction, and recombinant secreted frizzled-related protein 4 alters the progression of renal fibrosis. J Am Soc Nephrol 2005; 16:2373-2384.

47 Hwang I, Seo EY, Ha H: Wnt/beta-catenin signaling: a novel target for therapeutic intervention of fibrotic kidney disease. Arch Pharm Res 2009;32:1653-1662.

-48 Awazu M, Omori S, Ishikura K, Hida M, Fujita $\mathrm{H}$ : The lack of cyclin kinase inhibitor p27(Kip1) ameliorates progression of diabetic nephropathy. J Am Soc Nephrol 2003; 14:699-708.

49 Wolf G, Schanze A, Stahl RA, Shankland SJ, Amann K: P27(Kip1) knockout mice are protected from diabetic nephropathy: evidence for p27(Kip1) haplotype insufficiency. Kidney Int 2005;68:1583-1589.

50 Guo R, Liu L, Barajas L: RT-PCR study of the distribution of connexin 43 mRNA in the glomerulus and renal tubular segments. Am J Physiol 1998;275:R439-R447.
51 Suzuki C, Isaka Y, Takabatake Y, Tanaka H, Koike M, Shibata M, Uchiyama Y, Takahara S, Imai E: Participation of autophagy in renal ischemia/reperfusion injury. Biochem Biophys Res Commun 2008;368:100-106.

52 Inoue K, Kuwana H, Shimamura Y, Ogata K, Taniguchi Y, Kagawa T, Horino T, Takao T, Morita T, Sasaki S, Mizushima N, Terada Y: Cisplatin-induced macroautophagy occurs prior to apoptosis in proximal tubules in vivo. Clin Exp Nephrol 2010;14:112-122.

53 Yang C, Kaushal V, Shah SV, Kaushal GP: Autophagy is associated with apoptosis in cisplatin injury to renal tubular epithelial cells. Am J Physiol Renal Physiol 2008; 294:F777-F787.

54 Jiang M, Liu K, Luo J, Dong Z: Autophagy is a renoprotective mechanism during in vitro hypoxia and in vivo ischemia-reperfusion injury. Am J Pathol 2010;176:1181-1192.

55 Kimura T, Takabatake Y, Takahashi A, Kaimori JY, Matsui I, Namba T, Kitamura H, Niimura F, Matsusaka T, Soga T, Rakugi H, Isaka Y: Autophagy protects the proximal tubule from degeneration and acute ischemic injury. J Am Soc Nephrol 2011;22:902-913.

56 Jiang M, Wei Q, Dong G, Komatsu M, Su Y, Dong Z: Autophagy in proximal tubules protects against acute kidney injury. Kidney Int 2012;82:1271-1283.

57 Komatsu M, Waguri S, Koike M, Sou YS, Ueno T, Hara T, Mizushima N, Iwata J, Ezaki J, Murata S, Hamazaki J, Nishito Y, Iemura S, Natsume T, Yanagawa T, Uwayama J, Warabi E, Yoshida H, Ishii T, Kobayashi A, Yamamoto M, Yue Z, Uchiyama Y, Kominami E, Tanaka K: Homeostatic levels of p62 control cytoplasmic inclusion body formation in autophagy-deficient mice. Cell 2007; 131:1149-1163.

58 Cina DP, Onay T, Paltoo A, Li C, Maezawa Y, De Arteaga J, Jurisicova A, Quaggin SE: Inhibition of mTOR disrupts autophagic flux in podocytes. J Am Soc Nephrol 2012;23: 412-420.

59 Satriano J, Sharma K: Autophagy and metabolic changes in obesity-related chronic kidney disease. Nephrol Dial Transplant 2013; 28(suppl 4):iv29-iv36.

60 Bolisetty S, Traylor AM, Kim J, Joseph R, Ricart K, Landar A, Agarwal A: Heme oxygenase-1 inhibits renal tubular macroautophagy in acute kidney injury. J Am Soc Nephrol 2010;21:1702-1712.

61 He W, Tan R, Dai C, Li Y, Wang D, Hao S, Kahn M, Liu Y: Plasminogen activator inhibitor- 1 is a transcriptional target of the canonical pathway of Wnt/beta-catenin signaling. J Biol Chem 2010;285:24665-24675.

62 Backues SK, Klionsky DJ: Autophagy gets in on the regulatory act. J Mol Cell Biol 2011;3: 76-77. 
63 Gao C, Cao W, Bao L, Zuo W, Xie G, Cai T, Fu W, Zhang J, Wu W, Zhang X, Chen YG: Autophagy negatively regulates Wnt signalling by promoting Dishevelled degradation. Nat Cell Biol 2010;12:781-790.

-64 Satriano J, Mansoury H, Deng A, Sharma K, Vallon V, Blantz RC, Thomson SC: Transition of kidney tubule cells to a senescent phenotype in early experimental diabetes. Am J Physiol Cell Physiol 2010;299:C374-C380.

65 Allen RG, Tresini M, Keogh BP, Doggett DL, Cristofalo VJ: Differences in electron transport potential, antioxidant defenses, and oxidant generation in young and senescent fetal lung fibroblasts (WI-38). J Cell Physiol 1999;180:114-122.

-66 Sitte N, Merker K, von Zglinicki T, Grune T: Protein oxidation and degradation during proliferative senescence of human MRC-5 fibroblasts. Free Radic Biol Med 2000;28:701708.
67 Chondrogianni N, Stratford FL, Trougakos IP, Friguet B, Rivett AJ, Gonos ES: Central role of the proteasome in senescence and survival of human fibroblasts: induction of a senescence-like phenotype upon its inhibition and resistance to stress upon its activation. J Biol Chem 2003;278:28026-28037.

68 Kuilman T, Michaloglou C, Vredeveld LC, Douma S, van Doorn R, Desmet CJ, Aarden LA, Mooi WJ, Peeper DS: Oncogene-induced senescence relayed by an interleukindependent inflammatory network. Cell 2008;133:1019-1031.
69 Frippiat C, Chen QM, Zdanov S, Magalhaes JP, Remacle J, Toussaint O: Subcytotoxic $\mathrm{H}_{2} \mathrm{O}_{2}$ stress triggers a release of transforming growth factor-beta 1, which induces biomarkers of cellular senescence of human diploid fibroblasts. J Biol Chem 2001;276: 2531-2537.

70 Lempiäinen J, Finckenberg P, Levijoki J, Mervaala E: AMPK activator AICAR ameliorates ischemia reperfusion injury in the rat kidney. Br J Pharmacol 2012;166:1905-1915.

71 Thakar CV, Christianson A, Freyberg R, Almenoff P, Render ML: Incidence and outcomes of acute kidney injury in intensive care units: a Veterans Administration study. Crit Care Med 2009;37:2552-2558.

-72 Ryter SW, Choi AM: Heme oxygenase-1: redox regulation of a stress protein in lung and cell culture models. Antioxid Redox Signal 2005;7:80-91. 